\title{
Malignant Rhabdoid Tumors Originating Within and Outside the Central Nervous System Are Clinically and Molecularly Heterogeneous
}

\author{
Emilia M. Pinto ${ }^{1,}$, Dima Hamideh ${ }^{2,}$, Armita Bahrami ${ }^{1}$, Brent A. Orr ${ }^{1}$, Tong Lin ${ }^{3}$, Stanley \\ Pounds $^{3}$, Gerard P. Zambetti ${ }^{1}$, Alberto S. Pappo ${ }^{2,4}$, Amar Gajjar ${ }^{2,4}$, Sameer Agnihotri ${ }^{5}$, and \\ Alberto Broniscer ${ }^{2,4}$ \\ 1Department of Pathology, St. Jude Children's Research Hospital, 262 Danny Thomas Place, \\ Memphis, TN 38105 USA \\ 2Department of Oncology, St. Jude Children's Research Hospital, 262 Danny Thomas Place \\ Memphis, TN 38105 USA \\ 3Department of Biostatistics, St. Jude Children's Research Hospital, 262 Danny Thomas Place \\ Memphis, TN 38105 USA \\ ${ }^{4}$ Department of Pediatrics, University of Tennessee Health Science Center, 50 North Dunlap \\ Street, Memphis, TN 38103, USA \\ ${ }^{5}$ Department of Neurological Surgery, Children's Hospital of Pittsburgh,4401 Penn Ave Pittsburgh, \\ PA 15224
}

\section{Abstract}

Multifocal synchronous or metachronous atypical teratoid rhabdoid tumors (ATRTs) and noncentral nervous system malignant rhabdoid tumors (extra-CNS MRTs) are rare cancers. We reviewed the clinical and radiologic characteristics of affected patients seen at our institution. Genotyping and analysis of copy number abnormalities (CNAs) in SMARCB1 were performed in germline and tumor samples. Tumor samples underwent genome-wide DNA methylation and CNA analysis. The median age at diagnosis of 21 patients was 0.6 years. Two-thirds of ATRTs and extra-CNS MRTs were diagnosed synchronously. Although kidney tumors predominated, including two patients with bilateral involvement, at least $30 \%$ of cases lacked renal involvement. Histopathologic review confirmed MRTs in all cases and INI1 expression loss in all tumors tested. Fourteen (78\%) of 18 patients tested had heterozygous germline SMARCB1 abnormalities. At least one allelic SMARCB1 abnormality was confirmed in $81 \%$ and $88 \%$ of ATRTs and extraCNS MRTs, respectively. Unsupervised hierarchical clustering analysis of DNA methylation in 27 tumors and comparison to a reference group of 150 ATRTs classified the CNS tumors $(n=14)$ as sonic hedgehog (64\%), tyrosinase (21\%), and MYC (14\%). The MYC subgroup accounted for $85 \%$ of 13 extra-CNS MRTs. Of 16 paired ATRTs and extra-CNS MRTs, the tumors in 7 of 8 patients showed a different pattern of genome-wide DNA methylation and/or CNAs suggestive of

Corresponding author: Alberto Broniscer, MD 4401 Penn Ave Pittsburgh, PA 15224; Phone +1 412 692-5056, Fax +1 412 692-3412, alberto.broniscer@chp.edu.

These authors contributed equally to this manuscript 
non-clonal origin. CNS and extra-CNS tumors had an identical SMARCB1 amplification $(n=1)$ or very similar DNA methylation pattern $(n=1)$ suggestive of clonal origin. All patients died of tumor progression. The clinical and molecular characteristics of multifocal ATRTs and extra-CNS MRTs are heterogeneous with most patients harboring a cancer predisposition. Although independent tumor origin was confirmed in most cases, metastatic spread was also documented. The recognition of their distinct molecular characteristics is critical in selecting new biologic therapies against these deadly cancers.

\section{Keywords}

atypical teratoid rhabdoid tumor; children; DNA methylation; malignant rhabdoid tumor; multifocal

\section{Introduction}

Malignant rhabdoid tumors (MRTs), one of the deadliest pediatric solid cancers which predominantly affect young children, were first described as a variant of Wilms' tumor in the kidney [2]. MRTs can arise from any location in the body, including the central nervous system (CNS), in which case they are known as atypical teratoid rhabdoid tumors (ATRTs) $[6,24,25]$. ATRTs and extra-CNS MRTs are strongly associated with biallelic inactivation of the tumor-suppressor gene SMARCB1 [3, 31].

The occurrence of synchronous or metachronous multifocal ATRTs and extra-CNS MRTs has long been recognized although this is a rare and poorly understood phenomenon associated with a dismal prognosis [3-5, 8, 11, 26, 29, 32]. Most cases reported primary kidney and CNS tumors in patients with rhabdoid tumor predisposition syndrome type 1 (OMIM\#609322), an autosomal dominant disorder caused by heterozygous germline abnormalities of SMARCB1 [4, 9, 11, 21, 26]. However, involvement of other extra-CNS organs and lack of $S M A R C B 1$ germline abnormalities have also been reported $[9,14-16$, 21]. In addition, heterozygous germline abnormalities in SMARCA4, another member of the SWI/SNF complex, are associated with rhabdoid tumor predisposition type 2 (OMIM\#613325), a rare and aggressive autosomal dominant disorder that predominantly affects very young children [17].

Recent studies reported detailed genome-wide molecular characteristics in ATRTs and extraCNS MRTs [10, 16, 19, 20, 23, 30]. However, only a few studies reported limited molecular characteristics in synchronous or metachronous multifocal ATRTs and extra-CNS MRTs, particularly distinct patterns of biallelic SMARCB1 abnormalities [4, 11, 12, 15, 19, 26].

Here we report clinical, radiologic, and molecular characteristics in one of the largest cohorts to date of children with synchronous or metachronous multifocal ATRTs and extraCNS MRTs. Synchronous or metachronous multifocal ATRTs and extra-CNS MRTs represent a clinical and molecular heterogeneous group, which has critical importance for the surveillance and therapy of these patients. 


\section{Materials and Methods}

Following institutional review board approval, we retrospectively reviewed the clinical and radiologic characteristics of all patients diagnosed with synchronous or metachronous multifocal ATRTs and extra-CNS MRTs who underwent consultation or treatment at our institution from January 1, 1987 through January 1, 2017. Clinical data were collected for all patients including treatment information and outcome. All imaging studies were reviewed at our institution as part of patients' care. Imaging of all CNS tumors was re-reviewed by a senior neuro-oncologist (AB). Patients had CNS radiologic evaluation performed using MRIs except in 4 cases where only a computed tomography (CT) was obtained.

Genomic DNA was obtained from fresh-frozen $(n=4)$ or formalin-fixed paraffin-embedded (FFPE; $n=37$ ) tumor and germline samples following standard procedures. For FFPE samples, DNA was isolated from two 10- $\mu \mathrm{m}$ thick sections or scraped from four 5- $\mu \mathrm{m}$ thick unstained slides using DNA QIAamp FFPE Tissue Kit (Qiagen, Hilden, Germany) following the manufacturer's instructions. DNA was quantified using a Nanodrop ND-8000 spectrophotometer (Thermo Scientific, Rockford, IL).

\section{Multiplex Ligation-Dependent Probe Amplification (MLPA) Analysis of SMARCB1}

Deletions or amplifications of SMARCB1 were evaluated by using a commercial MLPA kit (Salsa P258 SMARCB1 probemix; MRC-Holland, Amsterdam, Netherlands). The probemix contains 18 probes for $S M A R C B 1$ (two probes for each exon) and 10 flanking probes surrounding the 22q11 and 22q12 chromosomal regions. The MLPA assay was carried out following the manufacturer's instructions and included five control samples. Analysis was performed using Coffalyzer software (MRC-Holland, Amsterdam, Netherlands).

\section{SMARCB1 Mutational Analysis}

Exons 1 through 9 of $S M A R C B 1$, including each flanking intron sequence, were amplified by polymerase-chain reaction (PCR). Sequencing of purified PCR products was performed on a high-throughput 3730xl DNA Analyzer (Applied Biosystems, Foster City, CA, USA). The sequence of individual exons was compared with the reported sequence in Ensembl database (ENSG00000099956). Primers and PCR conditions are detailed in Online Resource 1 .

\section{Illumina Infinium Human 850k Bead Array}

Prior to $\mathrm{CpG}$ methylation analysis, the quality of all DNA samples obtained from FFPE blocks was assessed using the Infinium FFPE QC Assay (Illumina, Inc., San Diego, CA). Quantitative PCR was used to compare 2ng of DNA against a standard sample provided by the manufacturer. The $\Delta \mathrm{Cq}$ value was calculated by subtracting the average value of $\mathrm{Cq}$ of the interrogated sample from the Cq value of the standard. All analyzed FFPE samples had a $\Delta \mathrm{Cq}<5$, which is the manufacturer's recommended threshold for suitability for FFPE restoration

DNA from FFPE (500ng) or from fresh-frozen samples (250-500ng) was treated with bisulfite using the Zymo EZ DNA Methylation Kit (Zymo Research, Irvine, CA) according 
to the following thermocycling conditions: 16 cycles at $95^{\circ} \mathrm{C}$ for $30 \mathrm{sec}$ and $50{ }^{\circ} \mathrm{C}$ for $1 \mathrm{hr}$. Following treatment with bisulfite, DNA samples were desulphonated, column purified, and eluted using $12 \mu \mathrm{L}$ of elution buffer (Zymo Research, Irvine, CA). For FFPE-derived samples, DNA was processed using the Illumina Infinium HD FFPE Restore kit (Illumina Inc., San Diego, CA) following the manufacturer's protocol. Bisulfite-converted DNA was then processed using the Illumina Infinium Methylation Assay including hybridization to HumanMethylation850k EPIC BeadChips (Illumina Inc., San Diego, CA), single-base extension assay, staining, and scanning using the Illumina HiScan system following the manufacturer's recommendations. Beta values representing the fraction of methylated cytosine present at each $\mathrm{CpG}$ site were calculated using the Illumina Genome Studio software (Illumina Inc., San Diego, CA) using the default settings. One tumor sample had been previously analyzed by Illumina Infinium Human 450k bead array (Illumina Inc., San Diego, CA) as previously described [20].

DNA methylation data analysis was performed using the open source statistical programming language $\mathrm{R}$ ( $\mathrm{R}$ Core Team, 2015). Raw data files generated by the iScan array scanner were read and pre-processed using minfi Bioconductor package [1]. With the minfi package, the same preprocessing steps as in Illumina's Genomestudio software were performed. In addition, we removed probes targeting the $\mathrm{X}$ and $\mathrm{Y}$ chromosomes, those containing-nucleotide polymorphism (dbSNP132 Common) within five base pairs of and including the targeted $\mathrm{CpG}$ site, and probes not mapping uniquely to the human reference genome (hg19) allowing for one mismatch. In total 395,401 probes common to both the Illumina 450k and EPIC arrays were kept for clustering analysis.

To determine the subgroup affiliation of our samples, we used a previously published dataset of DNA methylation in ATRTs available from the gene-expression omnibus (GSE70460) [20]. In this analysis, we combined our cases with 150 reference ATRT samples for unsupervised hierarchical clustering as previously described [20]. In brief, the 10,000 most variable methylated $\mathrm{CpG}$ probes measured by standard deviation across combined samples were selected. 1-Pearson correlation was calculated as the distance measured between samples and the unsupervised hierarchical clustering was performed by Ward's minimum variance agglomeration method. All samples analyzed could be assigned to one of the three previously described methylation subgroups [20]. We also performed consensus clustering analysis of the 10,000 most variable methylation probes selected by standard deviation across 176 samples (tumors included in the current manuscript and reference 20 minus one overlapping case). Consensus clustering was performed using the ConsensusClusterPlus package version 1.42 using the following non-default parameters: maximum $\mathrm{K}=5$, replication=1,000, pItem=0.9, distance="pearson", innerLinkage="ward.D".

Analysis of copy number abnormalities (CNAs) based on Illumina methylation arrays was performed using conumee Bioconductor package using default settings [18]. The combined intensities of all available $\mathrm{CpG}$ probes were normalized against control samples from normal brain tissue using a linear regression approach. Mean segment value of -0.2 and 0.2 were used as thresholds to call copy number loss and gain, respectively. 
The raw DNA methylation data were deposited at the NCBI Gene Expression Omnibus (GEO; accession number GSE107946).

\section{Results}

We identified 21 patients (11 [52\%] females) with multifocal ATRTs and extra-CNS MRTs (Table 1). The median age at diagnosis of first MRT was 0.6 years (range: 0 to 11.2). Tumor development was considered synchronous $(n=14$ [67\%]; median interval of 4 days between diagnoses; range: 0 to 21 ) or metachronous ( $n=7$; median interval of 247 days between diagnoses; range: 109 to 435) based on the interval from diagnosis of ATRT and extra-CNS MRTs. Fourteen (67\%) and seven (33\%) patients initially presented with signs and symptoms related to CNS and extra-CNS involvement, respectively (Table 1). Signs of increased intracranial pressure were reported in two-thirds of patients who presented with CNS-related symptoms. Other symptoms included seizures $(n=2 ; 14 \%)$, cranial nerve palsies $(n=4 ; 28 \%)$, and ataxia $(n=2)$. Tumor causing abdominal distension $(n=4 ; 57 \%)$ was the most common non-CNS-related symptom. Other non-CNS-related symptoms consisted of enlarging soft tissue masses $(n=2,28 \%)$ and hematuria $(n=1)$.

Primary location of ATRTs and extra-CNS tumors is described in Table 1. Two patients had CNS tumors with radiologic characteristics suggestive of meningeal origin (extra-axial tumors). All CNS tumors diagnosed by MRI ( $n=17)$ had radiologic features suggestive of aggressive cancers including a restricted apparent diffusion coefficient. Staging for CNS metastasis in 19 patients showed no disease spread (M0, $n=10)$, or metastatic disease in the brain (M2; $n=4)$ or spinal canal (M3; $n=5)$. Radiologic work-up for extra-CNS sites varied over time but all patients except one (Patient 21) underwent at least a CT of the chest, abdomen, and pelvis to characterize the primary tumor site and extent of involvement. Although tumors initially originated from the kidneys in 12 cases (57\%), including bilateral involvement $(n=2)$, other primary sites included soft tissues $(n=2,10 \%)$, musculoskeletal compartment, an axillary mass (lymph node), and bladder. (Table 1). Patient 10 had initially an enlarging superficial soft tissue mass and was subsequently diagnosed with a renal tumor. Staging for extra-CNS metastasis showed eight metastatic cases involving the lungs $(n=5)$, soft tissues $(n=4)$, and liver $(n=2)$.

Histopathologic review according to the most recent WHO criteria confirmed the diagnoses of MRT or ATRT in all tumors. Loss of INI1 expression in tumor cells by immunohistochemistry was confirmed in all samples tested (ATRT [ $n=14]$ and extra-CNS MRTs [ $n=17]$ ) (Table 2). Four and three ATRTs and extra-CNS tumors did not undergo histologic confirmation, respectively (Table 1).

Despite aggressive multi-modality therapy, all patients died of tumor progression after a median interval from diagnosis of 0.7 years (range: 0.1 to 4.5 years) (Table 1).

\section{Mutational Analysis of SMARCB1}

Analyses of SMARCB1 for CNAs and/or mutations were done in germline samples in all except for three patients (Table 2). These analyses were conducted from DNA obtained from peripheral-blood mononuclear cells $(n=10)$, from FFPE samples obtained from the normal 
kidney at the time of nephrectomy $(n=7)$, or from both $(n=1)$. Only $4(22 \%)$ of 18 patients lacked SMARCB1 germline abnormalities identified by Sanger sequencing and MLPA (Table 2). Germline abnormalities consisted of heterozygous mutations $(n=8)$ or deletions $(n=4)$, and large heterozygous losses of 22q11.23 (702 and 976kb) detected by commercially available microarrays.

The results of the same analyses in tumor samples are shown in Table 2. While biallelic and monoallelic SMARCB1 abnormalities were confirmed in $8(50 \%)$ and $5(31 \%)$ CNS tumors, respectively, they were demonstrated in 10 (59\%) and 5 (29\%) extra-CNS tumors, respectively. One patient (Patient 2) had the same focal amplification of exons 4 and 5 detected by MLPA in the CNS and extra-CNS tumors but not in the germline sample (Table 2).

\section{ATRTs and Extra-CNS MRT Display Distinct Methylation Profiles}

Unsupervised hierarchical clustering analysis of genome-wide DNA methylation was conducted in 27 tumors, 16 of them paired samples of CNS and extra-CNS tumors in the same patient (Figure 1 and 2). Among 16 paired samples, we observed two robust clusters, one where extra-CNS MRTs accounted for eight of nine samples, and a second group comprised only of ATRTs (Figure 2).

Subgroup assignment based on DNA methylation of CNS and extra-CNS tumors was for the most part quite distinct (Figures 1 and 2 and Table 2). ATRTs were classified as sonic hedgehog (SHH; $n=9[64 \%])$, tyrosinase ( $n=3[21 \%])$, and MYC ( $n=2[14 \%])$. Strikingly, $85 \%$ (11/13) of our extra-CNS MRTs clustered into the MYC subgroup (Figure 1).

Consensus clustering also supported the classification into three subgroups (data not provided). Only patients 6 and 7 shared the same subgroup assignment of ATRTs and extraCNS MRTs (Table 2). The separate ATRT in the lower thoracic spinal cord and the MRTs in the upper thoracic cavity of patient 7 had a very similar DNA methylation pattern (Figure 2 and Table 2).

CNA analyses are presented in Online Resource 2. Extra-CNS tumors had more copy gains and losses than ATRTs (Figure 3 and Online Resource 2). Multiple areas of recurrent focal chromosomal gains (e.g. chromosomes 2, 5, 6, 7, 12, 14, 15, and 17) were found mostly in extra-CNS MRTs, some of which contain genes associated with MRTs (e.g., HOTAIR and $H O X B$ cluster in chromosomes $12 \mathrm{q}$ and $17 \mathrm{q}$, respectively). Chromosome losses were less common (e.g., chromosomes 1, 14, and 22).

Like the DNA methylation analysis, the comparison of CNAs between paired ATRTs and extra-CNS MRTs in 8 patients showed a distinct pattern (Figure 3 and Online Resource 2).

\section{Discussion}

Although the occurrence of synchronous or metachronous multifocal MRTs was first reported more than 30 years ago [8], very little is known about these deadly cancers. The largest series to date reported 30 affected patients treated over five consecutive National Wilms' Tumor studies conducted throughout the US over 33 years [29]. The occurrence of 
synchronous or metachronous multifocal ATRTs and extra-CNS MRTs mostly in patients who harbored rhabdoid tumor predisposition syndrome type 1 and the documentation of distinct "second hits" in these tumors has led to the conclusion that this phenomenon was exclusively a result of multiple tumors independently arising in individuals with an autosomal dominant genetic disorder ("first hit") [4, 5, 9, 11, 22, 27]. On the other hand, other reports continued to attribute the involvement of multiple sites to metastatic tumor spread $[5,29,32]$.

We have shown for the first time the heterogeneity in clinical and molecular characteristics of synchronous or metachronous multifocal ATRTs and extra-CNS MRTs in the second largest series reported to date. Similar to data reported in multiple previous smaller studies $[3-5,8,26,29,32]$, the presentation of multifocal MRTs in our patients varied greatly based on the interval to develop the second tumor, age of affected subjects, and differences in CNS and extra-CNS primary sites. We also showed that at least $30 \%$ of extra-CNS MRTs may arise from sites outside the kidneys, which may be of practical importance for affected patients. Although there are currently no standard screening procedures for patients with rhabdoid tumor predisposition syndrome type 1 [13], very commonly only a renal ultrasound of the kidneys is recommended at regular intervals, which is an inadequate evaluation based on our findings. We ruled out rhabdoid tumor predisposition syndrome type 2 in all our patients except one (Patient 5) by confirming INI1 expression loss in tumor cells and/or biallelic SMARCB1 abnormalities in at least one of the MRTs.

To our knowledge, only one manuscript used multiple methods in addition to the analysis of biallelic $S M A R C B 1$ abnormalities to evaluate the mechanism of tumorigenesis of multifocal MRTs [15]. We report for the first time the methylation profile of a relatively large number of synchronous or metachronous multifocal ATRTs and extra-CNS MRTs. In contrast to recent studies [20,30], our findings that two-thirds of ATRTs clustered in the SHH group was surprising considering that their median age at diagnosis was only 0.7 years and that five $(56 \%)$ of them originated from the posterior fossa, both characteristics most commonly associated with the tyrosinase subgroup. As expected, the two tumors that clustered in the MYC subgroup originated in the spine $(n=1)$ or affected the oldest patient in this cohort $(n=1)$ [20, 30]. Significant differences in gene expression and DNA methylation analysis had been previously described between ATRTs and extra-CNS MRTs [7, 10, 20, 30]. We showed for the first time that these molecular differences are also present in most synchronous or metachronous multifocal ATRTs and extra-CNS MRTs, including paired samples (Figures 1 and 2, and Table 2). The molecular differences observed among multifocal MRTs arising in the same patient has potential clinical implications as pre-clinical studies showed that drug sensitivities in MRTs are dependent on tumor biology as determined by the DNA methylation subgroup assignment [20,30]. For example, platelet-derived growth factor receptor inhibitors showed promising activity against pre-clinical models of tyrosinase ATRTs but not against those of different subgroups [30]. Although we gathered a relatively large cohort of multifocal MRTs, the number of affected patients is still small. Therefore, our results will need to be validated in future larger studies.

The analysis of biallelic $S M A R C B 1$ abnormalities in our patients' tumor samples was not as informative as previously described since we did not perform SNP-based array analysis to 
detect copy number neutral loss of heterozygosity $[11,19]$. Fourteen $(78 \%)$ of our patients harbored heterozygous germline SMARCB1 abnormalities, seven (Patients 6, 7, 8, 9, 11, 12, and 19) of which had paired CNS and extra-CNS tumors available for gene sequencing, DNA methylation array and CNA analysis (Figures 1 and 3, and Table 2). Although five (71\%; patients 8, 9, 11, 12, and 19) of these seven paired tumors clustered in different methylation subgroups and had completely distinct patterns of CNAs, three (Patients 8, 9, and 12) had identical SMARCB1 abnormalities identified in both cancers. The differences in DNA methylation and CNA in these five cases, including the three with identical SMARCB1 abnormalities in both tumors, suggest a non-clonal origin in the context of rhabdoid tumor predisposition syndrome type 1 (Figure 2 and 3, Table 2, and Online Resource 2). Despite clustering to the same DNA methylation subgroup and sharing identical SMARCB1 abnormalities, the difference in DNA methylation profile and CNAs also suggest a non-clonal origin of both tumors in patient 6 . Only patient 7 had identical $S M A R C B 1$ abnormalities and a very similar pattern of DNA methylation comparing the ATRT and extra-CNS MRT to suggest that the later represented metastatic disease (Figures 2 and 3). In fact, the radiologic findings of this later patient were very suggestive of metastatic progression in the pelvis, abdomen, and chest because her spinal tumor had grown through the neural foramina and outside the CNS. The distribution of the DNA methylation subgroups between CNS and extra-CNS tumors was quite different among the seven remaining patients with a confirmed cancer predisposition, but the lack of molecular analyses in paired samples prevented us from drawing further conclusions (Figure 1).

Among the remaining patients evaluated in the current study, DNA methylation and CNA analysis of paired tumors in patient 15, who lacked SMARCB1 germline abnormalities, showed a distinct pattern. Interestingly, both his CNS and extra-CNS tumor shared the same truncating mutation. Although we cannot completely rule out metastatic tumor spread as a possible mechanism for the formation of multifocal tumors with identical SMARCB1 mutations, this hypothesis seems unlikely in view of the distinct pattern of DNA methylation and CNAs. Instead, we feel that the molecular characteristics described above are more suggestive of a SMARCB1 somatic mosaicism leading to non-clonal multifocal MRTs formation. Unfortunately, no further testing was performed when this patient was still alive to confirm this hypothesis. The finding of the same $S M A R C B 1$ amplification in the ATRT and extra-CNS MRT in patient 2, who also lacked a germline SMARCB1 abnormality, suggests that those tumors shared a clonal origin and that the CNS spread represented a late metastasis. This area of amplification could not be confirmed by the DNA methylation array because of the absence of $\mathrm{CpG}$ probes in the affected exons

Despite the relatively large cohort of affected individuals and extensive molecular analyses, our study had some limitations inherent to retrospective studies, including the unavailability of adequate tumor and germline samples for analyses and lack of homogeneous imaging and genetic work-up for all cases. Although the imaging modalities varied over time, all patients underwent radiologic work-up to assess at least the involvement of CNS and both kidneys at first diagnosis of MRT. No histologic confirmation of second MRTs was obtained in seven cases, including patients with overt (e.g., patient 14 with bilateral kidney involvement) or subtle presentations (e.g., patient 16 with bladder tumor) (Online Resources 3-5). Three of these seven patients underwent at least one attempt for histologic confirmation which was 
unsuccessful, which reflects some of the obstacles in properly evaluating these patients. Finally, we were unable to perform more detailed molecular studies including genome-wide sequencing of tumor samples and analysis of SMARCB1 introns in germline and tumor samples, the later probably accounting for the lack of genetic abnormalities in some cases [28].

In conclusion, we showed that children with synchronous or metachronous multifocal ATRTs and extra-CNS MRTs, most of which are associated with rhabdoid tumor predisposition syndrome type 1 , have heterogeneous clinical and molecular characteristics. Although non-clonal independent tumorigenesis of these multiple tumors in patients with a cancer predisposition likely accounts for most cases, metastatic spread can also occur. In cases of multifocal MRTs, it is critical that families receive thorough genetic counseling and that proper genetic testing is offered. Despite the common use of intensive chemotherapy with or without radiation therapy, all our patients died of their tumors within a short interval. Based on our results, the incorporation of early palliative measures in the care of young patients with multifocal MRTs is a reasonable option. We hope that our findings will influence the treatment of patients with these deadly cancers.

\section{Supplementary Material}

Refer to Web version on PubMed Central for supplementary material.

\section{Acknowledgments}

This work was supported by the United States National Institutes of Health Cancer Center Support (CORE) Grant P30 CA21765 and by the American Lebanese Syrian Associated Charities (ALSAC). We thank Geoffrey Neale, $\mathrm{PhD}$ and Emily Walker for assistance in performing the DNA methylation studies.

\section{References}

1. Aryee MJ, Jaffe AE, Corrada-Bravo H, Ladd-Acosta C, Feinberg AP, Hansen KD, Irizarry RA. Minfi: a flexible and comprehensive Bioconductor package for the analysis of Infinium DNA methylation microarrays. Bioinformatics. 2014; 30:1363-1369. DOI: 10.1093/bioinformatics/ btu049 [PubMed: 24478339]

2. Beckwith JB, Palmer NF. Histopathology and prognosis of Wilms tumor: results from the First National Wilms' Tumor Study. Cancer. 1978; 41:1937-1948. DOI:

10.1002/1097-0142(197805)41:5<1937::AID-CNCR2820410538>3.0.CO;2-U [PubMed: 206343]

3. Biegel JA, Zhou JY, Rorke LB, Stenstrom C, Wainwright LM, Fogelgren B. Germ-line and acquired mutations of INI1 in atypical teratoid and rhabdoid tumors. Cancer Res. 1999; 59:74-79. [PubMed: 9892189]

4. Biegel JA, Fogelgren B, Wainwright LM, Zhou JY, Bevan H, Rorke LB. Germline INI1 mutation in a patient with a central nervous system atypical teratoid tumor and renal rhabdoid tumor. Genes Chromosomes Cancer. 2000; 28:31-37. DOI: 10.1002/(SICI)1098-2264(200005)28:1<31::AIDGCC4>3.0.CO;2-Y [PubMed: 10738300]

5. Biegel JA, Tan L, Zhang F, Wainwright L, Russo P, Rorke LB. Alterations of the hSNF5/INI1 gene in central nervous system atypical teratoid/rhabdoid tumors and renal and extrarenal rhabdoid tumors. Clin Can Res. 2002; 8:3461-3467.

6. Biggs PJ, Garen PD, Powers JM, Garvin AJ. Malignant rhabdoid tumor of the central nervous system. Hum Pathol. 1987; 18:332-337. doi: org/10.1016/S0046-8177(87)80161-2. [PubMed: 3030922] 
7. Birks DK, Donson AM, Patel PR, Sufit A, Algar EM, Dunham C, Kleinschmidt-DeMasters BK, Handler MH, Vibhakar R, Foreman NK. Pediatric rhabdoid tumors of kidney and brain show many differences in gene expression but share dysregulation of cell cycle and epigenetic effector genes. Pediatr Blood Cancer. 2013; 60:1095-1102. DOI: 10.1002/pbc.24481 [PubMed: 23382118]

8. Bonnin JM, Rubinstein LJ, Palmer NF, Beckwith JB. The association of embryonal tumors originating in the kidney and in the brain. A report of seven cases. Cancer. 1984; 54:2137-2146. DOI: 10.1002/1097-0142(19841115)54:10<2137::AID-CNCR2820541014>3.0.CO;2-D [PubMed: 6091860]

9. Bourdeaut F, Lequin D, Brugières L, et al. Frequent hSNF5/INI1 germline mutations in patients with rhabdoid tumor. Clin Cancer Res. 2011; 17:31-38. DOI: 10.1158/1078-0432.CCR-10-1795 [PubMed: 21208904]

10. Chun HJ, Lim EL, Heravi-Moussavi A, et al. Genome-wide profiles of extra-cranial malignant rhabdoid tumors reveal heterogeneity and dysregulated developmental pathways. Cancer Cell. 2016; 29:394-406. DOI: 10.1016/j.ccell.2016.02.009 [PubMed: 26977886]

11. Eaton KW, Tooke LS, Wainwright LM, Judkins AR, Biegel JA. Spectrum of SMARCB1/INI1 mutations in familial and sporadic rhabdoid tumors. Pediatr Blood Cancer. 2011; 56:7-15. DOI: 10.1002/pbc.22831 [PubMed: 21108436]

12. Fort DW, Tonk VS, Tomlinson GE, Timmons CF, Schneider NR. Rhabdoid tumor of the kidney with primitive neuroectodermal tumor of the central nervous system: associated tumors with different histologic, cytogenetic, and molecular findings. Genes Chromosomes Cancer. 1994; 11:146-152. DOI: 10.1002/gcc.2870110303 [PubMed: 7530483]

13. Foulkes WD, Kamihara J, Evans DGR, Brugières L, Bourdeaut F, Molenaar JJ, Walsh MF, Brodeur GM, Diller L. Cancer surveillance in Gorlin syndrome and rhabdoid tumor predisposition syndrome. Clin Cancer Res. 2017; 23:e62-e67. DOI: 10.1158/1078-0432.CCR-17-0595 [PubMed: 28620006]

14. Fujita M, Sato M, Nakamura M, et al. Multicentric atypical teratoid/rhabdoid tumors occurring in the eye and fourth ventricle of an infant: case report. J Neurosurg. 102:299-302.

15. Giunti L, Bernini G, Forni M, Tucci F, Wheeler E, Sardi I. Clonality analysis of pediatric multiple tumors: two case reports and laboratory investigation. J Pediatr Hematol Oncol. 2006; 28:241-248. DOI: 10.1097/01.mph.0000212909.91770.72 [PubMed: 16679923]

16. Hasselblatt M, Isken S, Linge A, et al. High-resolution genomic analysis suggests the absence of recurrent genomic alterations other than SMARCB1 aberrations in atypical teratoid/rhabdoid tumors. Genes Chromosomes Cancer. 2013; 52:185-190. DOI: 10.1002/gcc.22018 [PubMed: 23074045]

17. Hasselblatt M, Nagel I, Oyen F, et al. SMARCA4-mutated atypical teratoid/rhabdoid tumors are associated with inherited germline alterations and poor prognosis. Acta Neuropathol. 2014; 128:453-456. DOI: 10.1007/s00401-014-1323-x [PubMed: 25060813]

18. HovestadtV, , ZapatkaM. conumee: Enhanced copy-number variation analysis using Illumina 450k methylation arrays. $\mathrm{R}$ package version 0.99.4. Bioconductor: open source software for bioinformaticshttp://www.bioconductor.org/packages/release/bioc/html/conumee.html

19. Jackson EM, Sievert AJ, Gai X, Hakonarson H, Judkins AR, Tooke L, Perin JC, Xie H, Shaikh TH, Biegel JA. Genomic analysis using high-density single nucleotide polymorphism-based oligonucleotide arrays and multiplex ligation-dependent probe amplification provides a comprehensive analysis of INI1/SMARCB1 in malignant rhabdoid tumors. Clin Cancer Res. 2009; 15:1923-1930. DOI: 10.1158/1078-0432.CCR-08-2091 [PubMed: 19276269]

20. Johann PD, Erkek S, Zapatka M, et al. Atypical teratoid/rhabdoid tumors are comprised of three epigenetic subgroups with distinct enhancer landscapes. Cancer Cell. 2016; 29:379-393. DOI: 10.1016/j.ccell.2016.02.001 [PubMed: 26923874]

21. Kordes U, Gesk S, Frühwald MC, et al. Clinical and molecular features in patients with atypical teratoid rhabdoid tumor or malignant rhabdoid tumor. Genes Chromosomes Cancer. 2010; 49:176181. DOI: 10.1002/gcc.20729 [PubMed: 19902524]

22. Kusafuka T, Miao J, Yoneda A, Kuroda S, Fukuzawa M. Novel germ-line deletion of SNF5/INI1/ SMARCB1 gene in neonate presenting with congenital malignant rhabdoid tumor of kidney and brain primitive neuroectodermal tumor. Genes Chromosomes Cancer. 2004; 40:133-139. DOI: 10.1002/gcc.20026 [PubMed: 15101046] 
23. Lee RS, Stewart C, Carter SL, et al. A remarkably simple genome underlies highly malignant pediatric rhabdoid cancers. J Clin Invest. 2012; 122:2983-2988. DOI: 10.1172/JCI64400 [PubMed: 22797305]

24. Parham DM, Weeks DA, Beckwith JB. The clinicopathologic spectrum of putative extrarenal rhabdoid tumors. An analysis of 42 Cases studied with immunohistochemistry or electron microscopy. Am J Surg Pathol. 1994; 18:1010-1029. [PubMed: 8092393]

25. Rorke LB, Packer RJ, Biegel JA. Central nervous system atypical teratoid/rhabdoid tumors of infancy and childhood: definition of an entity. J Neurosurg. 1996; 85:56-65. [PubMed: 8683283]

26. Savla J, Chen TT, Schneider NR, Timmons CF, Delattre O, Tomlinson GE. Mutations of the hSNF5/INI1 gene in renal rhabdoid tumors with second primary brain tumors. J Natl Cancer Inst. 2000; 92:648-650. DOI: 10.1093/jnci/92.8.648 [PubMed: 10772683]

27. Sévenet N, Sheridan E, Amram D, Schneider P, Handgretinger R, Delattre O. Constitutional mutations of the hSNF5/INI1 gene predispose to a variety of cancers. Am J Hum Genet. 1999; 65:1342-1348. DOI: 10.1086/302639 [PubMed: 10521299]

28. Tauziède-Espariat A, Masliah-Planchon J, Brugières L, et al. Deep intronic hotspot variant unraveling rhabdoid tumor predisposition syndrome in two patients with atypical teratoid and rhabdoid tumor. Eur J Hum Genet. 2017; in press. doi: 10.1038/ejhg.2017.115

29. Tomlinson GE, Breslow NE, Dome J, et al. Rhabdoid tumor of the kidney in the National Wilms' Tumor Study: age at diagnosis as a prognostic factor. J Clin Oncol. 2005; 23:7641-7645. DOI: 10.1200/JCO.2004.00.8110 [PubMed: 16234525]

30. Torchia J, Picard D, Lafay-Cousin L, et al. Molecular subgroups of atypical teratoid rhabdoid tumours in children: an integrated genomic and clinicopathological analysis. Lancet Oncol. 2015; 16:569-582. DOI: 10.1016/S1470-2045(15)70114-2 [PubMed: 25882982]

31. Versteege I, Sévenet N, Lange J, Rousseau-Merck MF, Ambros P, Handgretinger R, Aurias A, Delattre O. Truncating mutations of hSNF5/INI1 in aggressive paediatric cancer. Nature. 1998; 394:203-206. DOI: 10.1038/28212 [PubMed: 9671307]

32. Weeks DA, Beckwith JB, Mierau GW, Luckey DW. Rhabdoid tumor of kidney. A report of 111 cases from the National Wilms' Tumor Study Pathology Center. Am J Surg Pathol. 1989; 13:439458. DOI: 10.1097/00000478-198906000-00001 [PubMed: 2543225] 


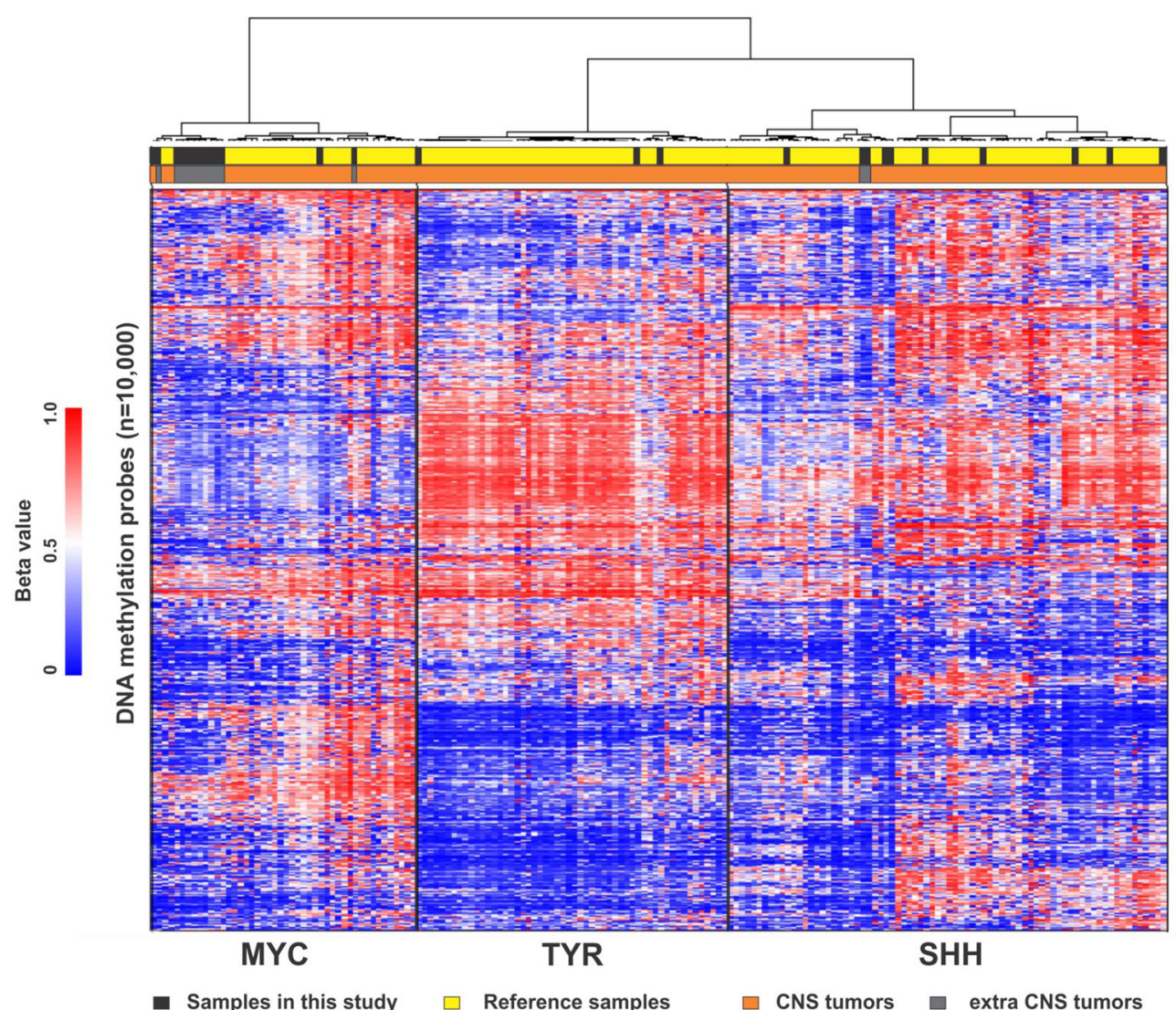

Fig 1.

Unsupervised hierarchical clustering analysis of genome-wide DNA methylation in 27 central nervous system (CNS; $n=14)$ and extra-CNS tumors $(n=13)$ compared to 150 previously published atypical teratoid rhabdoid tumors 


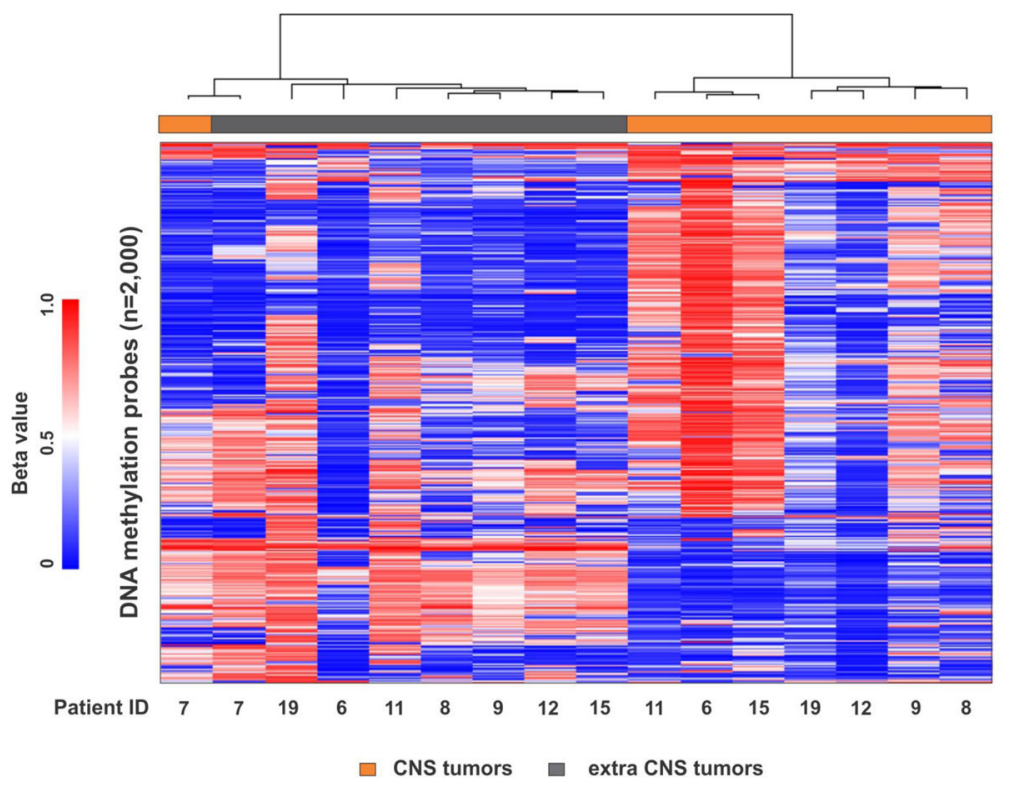

Fig 2.

Unsupervised hierarchical clustering analysis of 16 paired central nervous system (CNS) and extra-CNS tumors in 8 patients 


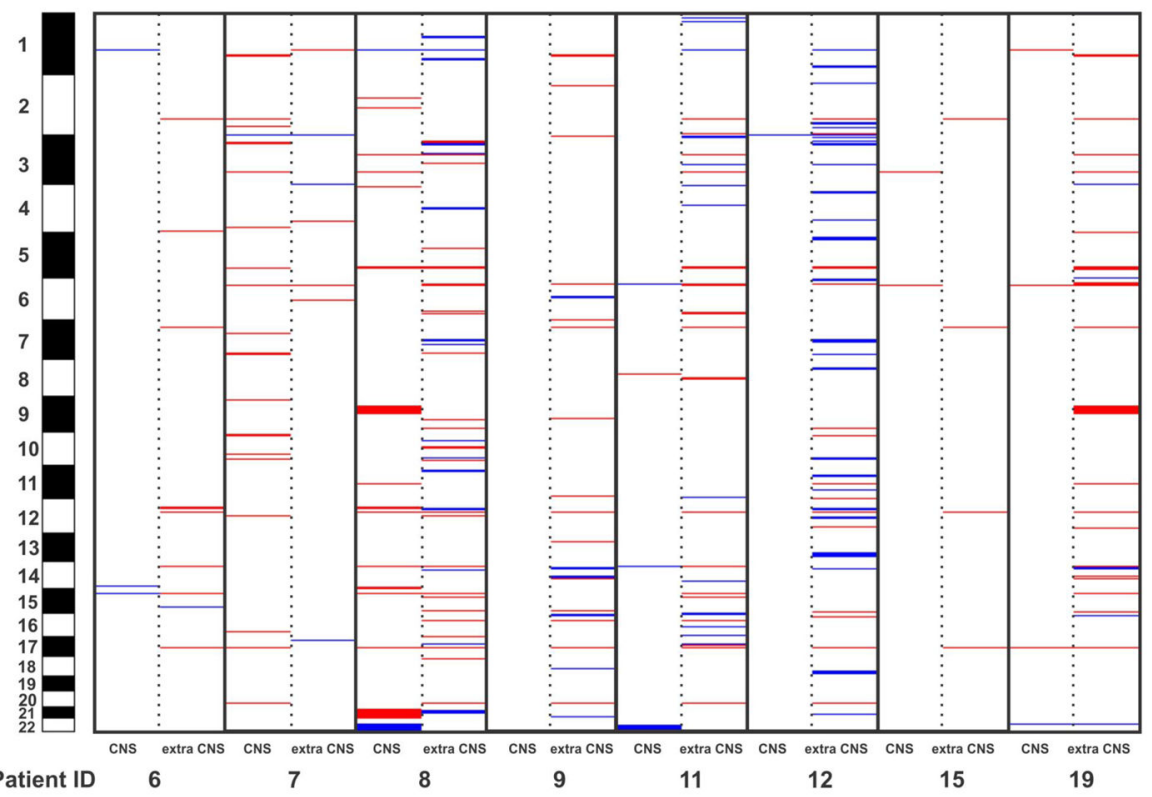

Fig 3.

Pattern of chromosomal gains (red) and losses (blue) in the entire genome except for sex chromosomes of 16 paired central nervous system (CNS) and extra-CNS tumors. The 22 non-sex chromosomes are displayed on the left vertical axis 


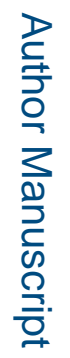
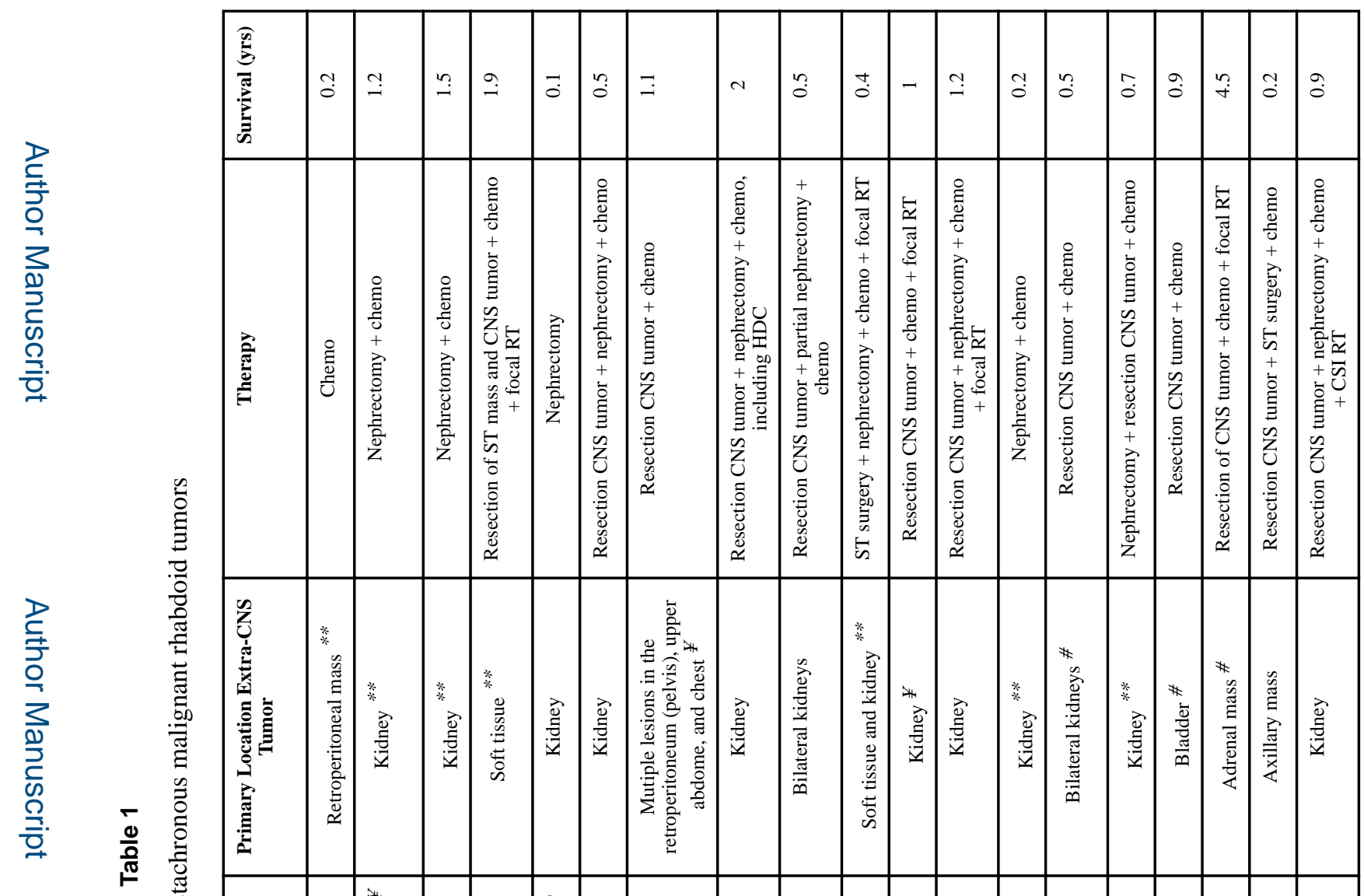

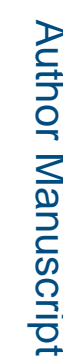

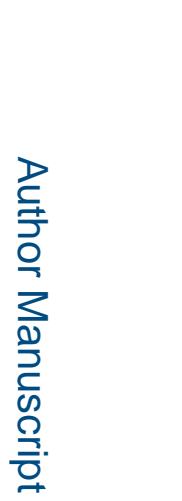

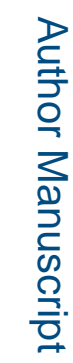
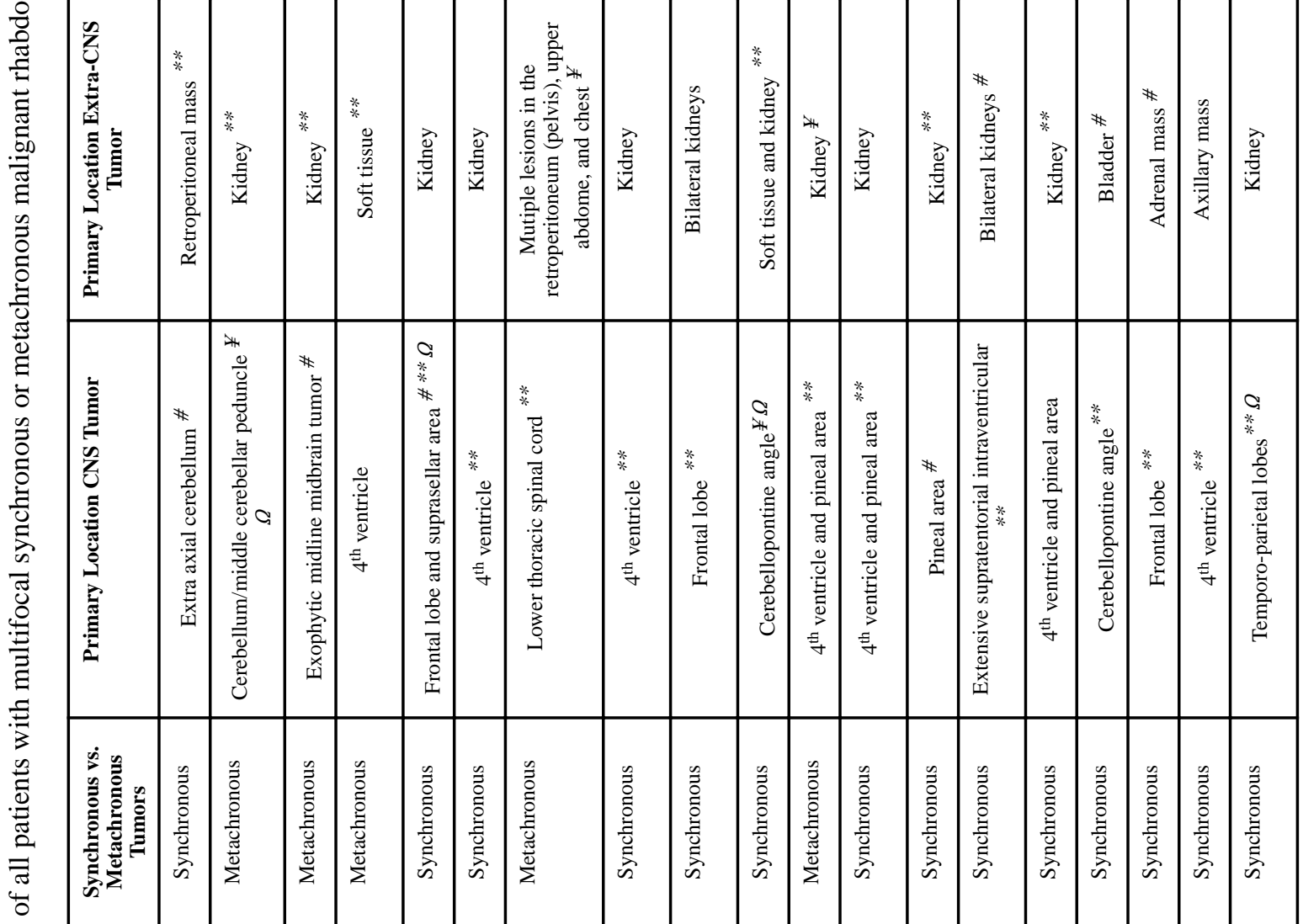

:

\begin{tabular}{|c|c|c|c|c|c|c|c|c|c|c|c|c|c|c|c|c|c|c|c|}
\hline 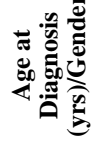 & 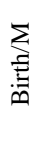 & 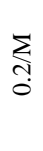 & 崩 & 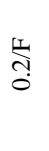 & ্ָ] & ल⿸厂 & 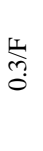 & $\sum_{i=1}^{n}$ & $\begin{array}{l}\frac{1}{n} \\
\vdots \\
0\end{array}$ & $\begin{array}{l}\Sigma \\
\text { L } \\
0\end{array}$ & 峁 & $\underset{i}{\stackrel{\Sigma}{i}}$ & 站 & $\begin{array}{l}\frac{1}{5} \\
\text { S. }\end{array}$ & $\underset{\substack{\text { I } \\
\text { In }}}{ }$ & $\underset{j}{\sum_{j}}$ & స્ & 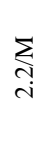 & $\underset{\sim}{\stackrel{\mid}{m}}$ \\
\hline 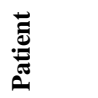 & - & $N$ & $m$ & $\nabla$ & in & 6 & $r$ & $\infty$ & $a$ & $\Theta$ & $=$ & $\simeq$ & 2 & $\Xi$ & 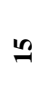 & $\stackrel{\varrho}{2}$ & $=$ & $\stackrel{\infty}{\sim}$ & 2 \\
\hline
\end{tabular}

Acta Neuropathol. Author manuscript; available in PMC 2019 August 01. 


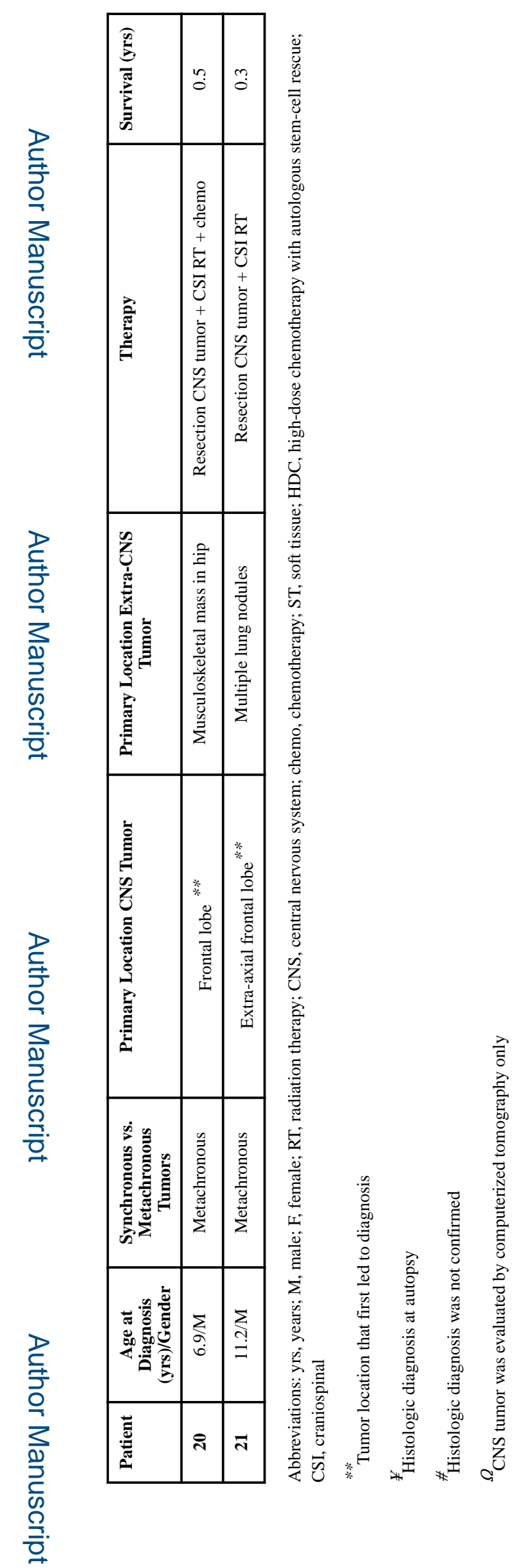

Acta Neuropathol. Author manuscript; available in PMC 2019 August 01. 


\begin{tabular}{|c|c|c|c|c|c|c|c|c|c|c|c|c|c|c|c|c|c|c|}
\hline 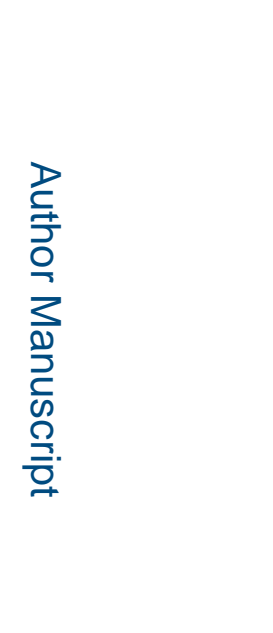 & & 总 & 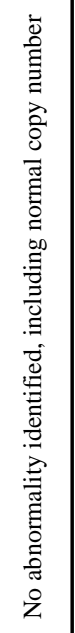 & 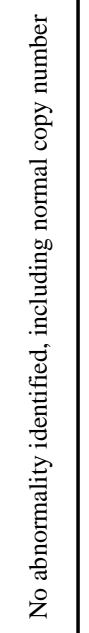 & 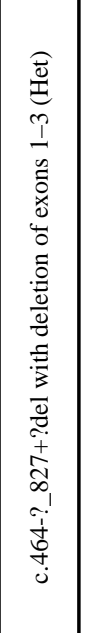 & 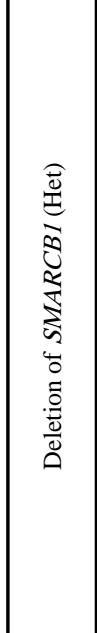 & $\frac{\pi}{z}$ & 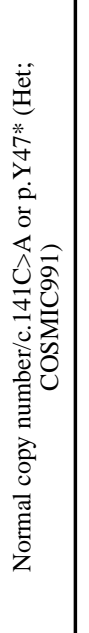 & 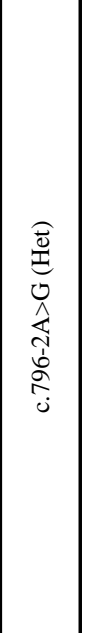 & 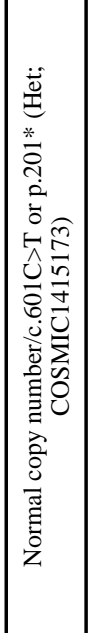 & 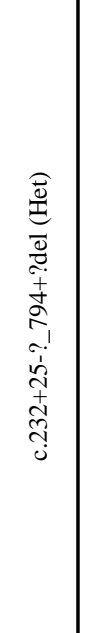 & 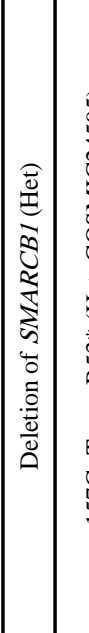 & 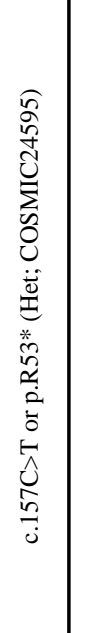 & 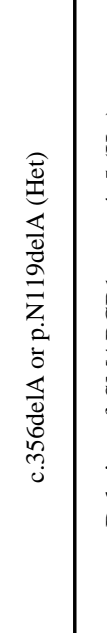 & 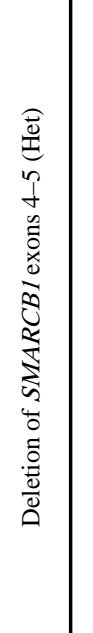 & 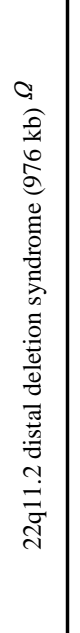 & 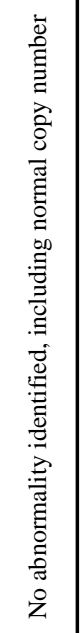 & 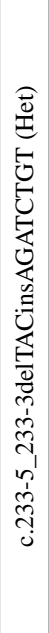 \\
\hline & & 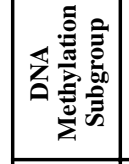 & $\mathbb{z}$ & 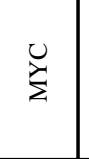 & 焉 & $\mathbb{z}$ & $\mid \overrightarrow{\vec{z}}$ & 焉 & $\stackrel{\circlearrowright}{\grave{\Sigma}}$ & 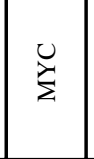 & $\begin{array}{l}\breve{\Xi} \\
\grave{\Sigma}\end{array}$ & 崶 & 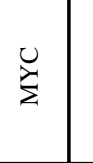 & $\stackrel{U}{\vec{z}}$ & 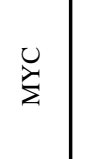 & $\frac{\pi}{z}$ & $\breve{\Xi}$ & $\tilde{z}$ \\
\hline 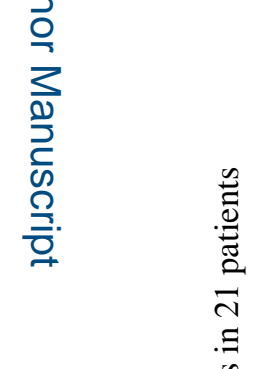 & 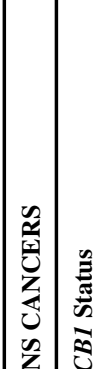 & 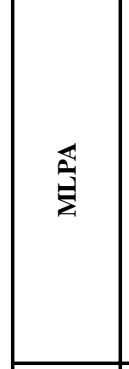 & 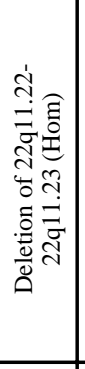 & 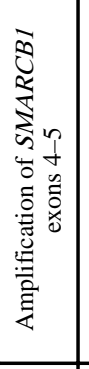 & 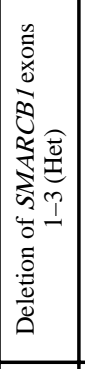 & 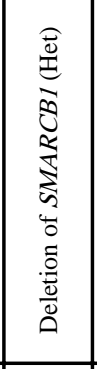 & 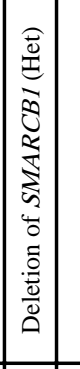 & 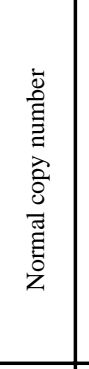 & 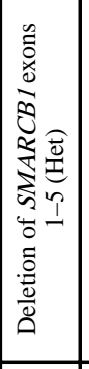 & 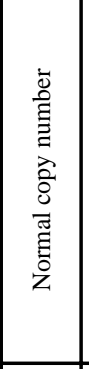 & 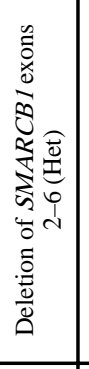 & 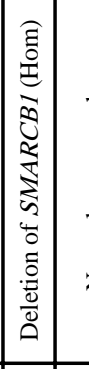 & 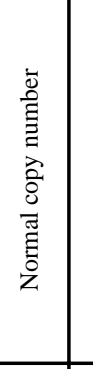 & 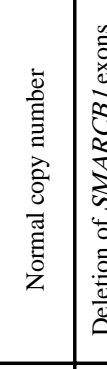 & 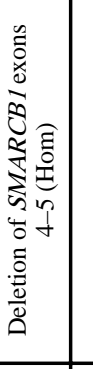 & $\overleftarrow{z}$ & 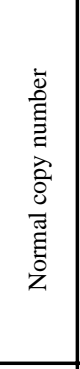 & $\mathbb{z}$ \\
\hline 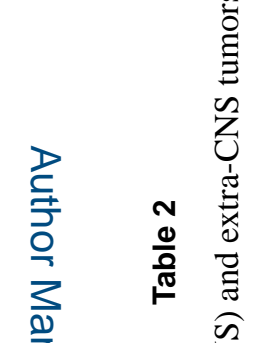 & 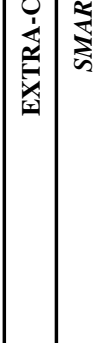 & 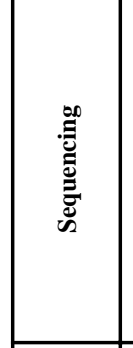 & 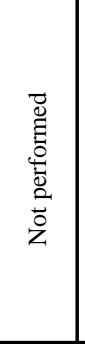 & 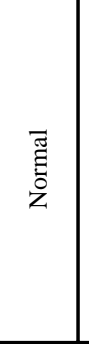 & 嵒 & 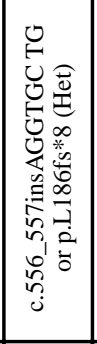 & $\frac{\pi}{z}$ & 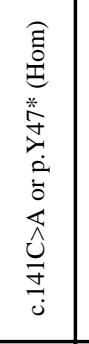 & 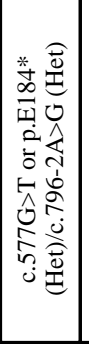 & 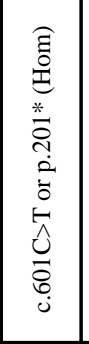 & 嵒 & 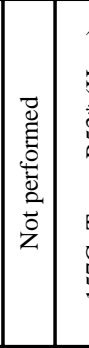 & 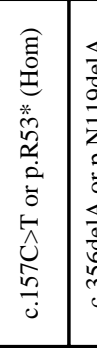 & 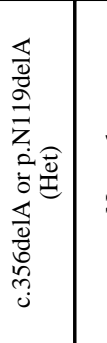 & 㤧 & z & 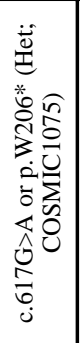 & $\bar{z}$ \\
\hline 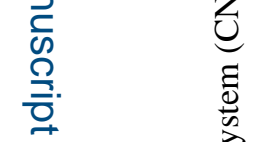 & & 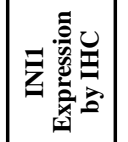 & 童 & 参 & 总 & 参 & $\mathbb{z}$ & 参 & 参 & 参 & 参 & 总 & 参 & 商 & 参 & $\mathbb{z}$ & 岁 & $\underline{z}$ \\
\hline 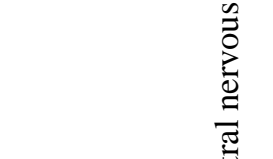 & & 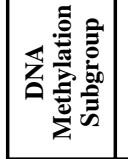 & $\mathbb{z}$ & 焉 & $\mathbb{z}$ & 畓 & $\mathbb{z}$ & 焉 & 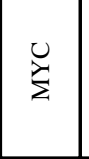 & $\stackrel{\mathscr{2}}{\ddot{t}}$ & 焉 & $\vec{z}$ & 焉 & 焉 & $\mathbb{z}$ & 焉 & 焉 & $\stackrel{2}{2}$ \\
\hline $\begin{array}{l}\bar{\Xi} \\
0 \\
0 \\
0 \\
\overline{0} \\
\bar{\Xi} \\
\tilde{\Xi} \\
\bar{\Xi} \\
0 \\
0 \\
0 \\
0\end{array}$ & 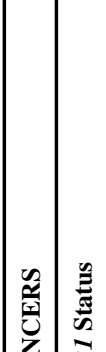 & 全 & $\frac{\pi}{z}$ & 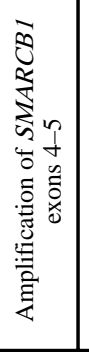 & $\frac{\pi}{z}$ & 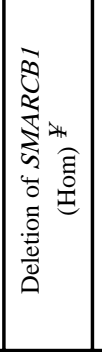 & $\frac{\pi}{z}$ & 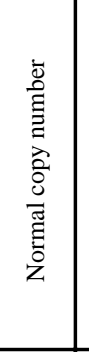 & 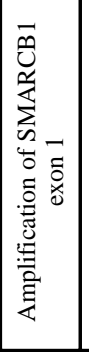 & 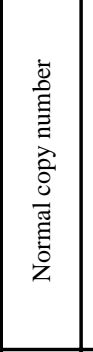 & 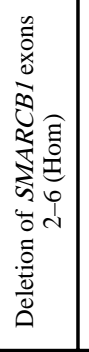 & 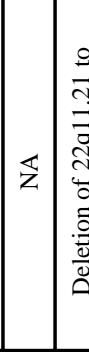 & 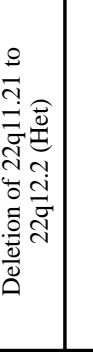 & 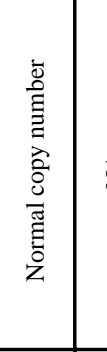 & 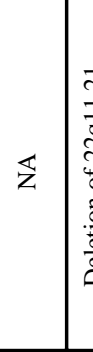 & 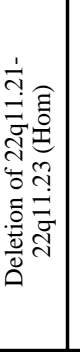 & 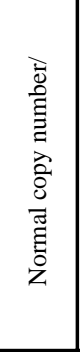 & 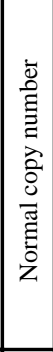 \\
\hline 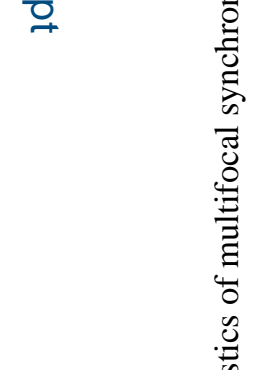 & 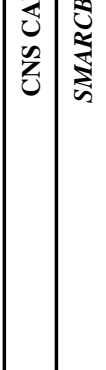 & 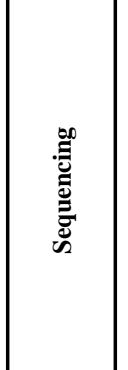 & $\S$ & $\frac{\pi}{z}$ & $\mathbb{z}$ & 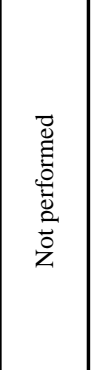 & $\underline{z}$ & 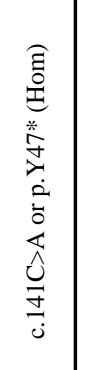 & 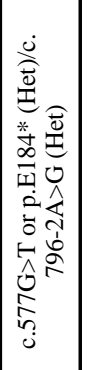 & 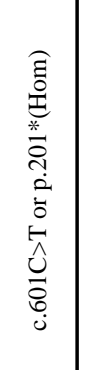 & 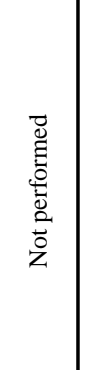 & $\mathbb{z}$ & 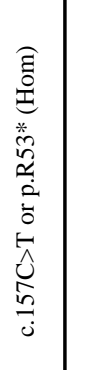 & 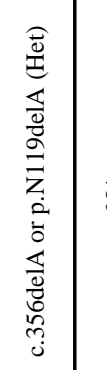 & $\widehat{z}$ & 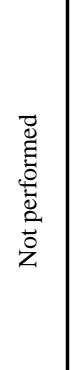 & 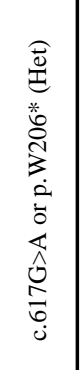 & 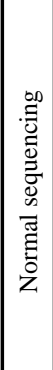 \\
\hline 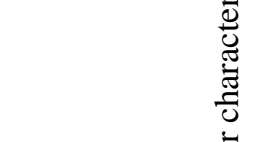 & & 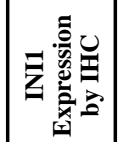 & $\mathbb{z}$ & 商 & $\mathbb{z}$ & 参 & $\mathbb{z}$ & 咅 & 参 & 参 & 参 & $|\vec{z}|$. & $\stackrel{3}{9}$ & $\tilde{z}$ & $\overleftarrow{z}$ & $\stackrel{\mathscr{3}}{9}$ & 参 & $\mid \begin{array}{l}0 \\
\vdots \\
\vdots \\
\vdots\end{array}$ \\
\hline $\begin{array}{l}\overline{\bar{U}} \\
\frac{\tilde{D}}{0} \\
\sum_{\bar{z}}\end{array}$ & & 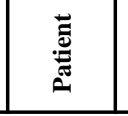 & - & $\sim$ & $m$ & $\sigma$ & in & 0 & $r$ & $\infty$ & $\sigma$ & 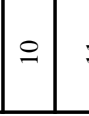 & $=$ & $\simeq$ & 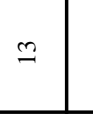 & \pm & 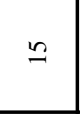 & 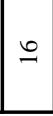 \\
\hline
\end{tabular}




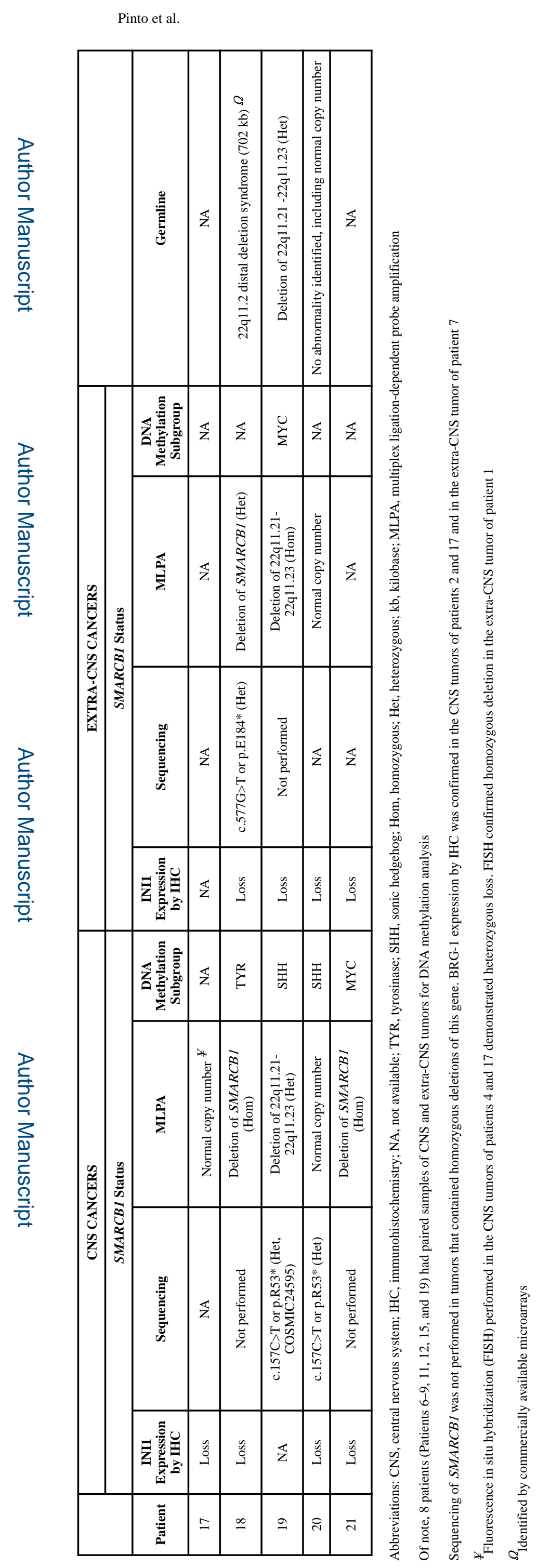

Acta Neuropathol. Author manuscript; available in PMC 2019 August 01. 